\title{
Giant Magnetoresistance in Organic Spin Valves
}

\author{
Dali Sun, ${ }^{1,2}$ Lifeng Yin, ${ }^{1,3}$ Chengjun Sun, ${ }^{1}$ Hangwen Guo, ${ }^{1}$ Zheng Gai, ${ }^{1,4}$ X.-G. Zhang, ${ }^{4,5}$ T. Z. Ward, ${ }^{1}$ \\ Zhaohua Cheng, ${ }^{2}$ and Jian Shen ${ }^{1,3, *}$ \\ ${ }^{1}$ Materials Science and Technology Division, Oak Ridge National Laboratory, Oak Ridge, Tennessee 37831, USA \\ ${ }^{2}$ State Key Laboratory of Magnetism and Beijing National Laboratory for Condensed Matter Physics, Institute of Physics, \\ Chinese Academy of Sciences, Beijing 100190, China \\ ${ }^{3}$ Department of Physics, Fudan University, Shanghai 200433, China \\ ${ }^{4}$ Center for Nanophase Materials Sciences, Oak Ridge National Laboratory, Oak Ridge, Tennessee 37831, USA \\ ${ }^{5}$ Computer Science and Mathematics Division, Oak Ridge National Laboratory, Oak Ridge, Tennessee 37831, USA
}

(Received 12 February 2010; published 11 June 2010)

\begin{abstract}
Interfacial diffusion between magnetic electrodes and organic spacer layers is a serious problem in the organic spintronics which complicates attempts to understand the spin-dependent transport mechanism and hurts the achievement of a desirably high magnetoresistance (MR). We deposit nanodots instead of atoms onto the organic layer using buffer layer assist growth. Spin valves using this method exhibit a sharper interface and a giant MR of up to $\sim 300 \%$. Analysis of the current-voltage characteristics indicates that the spin-dependent carrier injection correlates with the observed MR.
\end{abstract}

For spintronics applications, organic spin valves are particularly attractive because of its flexibility and low cost. A critical issue in the evolution of these devices is the need to achieve high magnetoresistance (MR) using a conventional trilayer base structure consisting of an organic layer sandwiched between two magnetic layers serving as magnetic electrodes. Using tris-(8hydroxyquinoline) aluminum $\left(\mathrm{Alq}_{3}\right)$ as the spacer layer between ferromagnetic $\mathrm{La}_{0.67} \mathrm{Sr}_{0.33} \mathrm{MnO}_{3}$ (LSMO) and cobalt electrodes, Xiong et al. measured a giant negative magnetoresistance of $40 \%$ at $11 \mathrm{~K}$ [1]. This initial work generated considerable interest in studying various types of organic spin valves aiming at both improving the magnetoresistance and understanding the underlying mechanism [2-7]. These efforts, however, have resulted in limited success in improving MR while generating a great deal of controversy related to the sign [1-4] and the mechanism [1,4-7] of MR. It has been generally agreed that these difficulties are largely the result of metal penetration from the top magnetic electrode into the soft organic layer-leading to a so-called ill-defined organic spacer layer $[1,2]$. It was previously shown that the influence of the ill-defined layer can be reduced by inserting an insulating layer (e.g., $\mathrm{Al}_{2} \mathrm{O}_{3}$ ) between the bottom electrode and the organic spacer layer [8-10]. However, the addition of an insulating barrier layer does not appear to greatly improve the MR effect while, at the same time, introducing new complications that might impede attempts to understand the fundamental mechanisms of MR in organic spin valves [11,12].

Because of their greater size, magnetic nanodots are expected to have a much lower diffusion rate into the organic spacer layer than individual adatoms as has been demonstrated with $\mathrm{Al}$ aggregation on $\mathrm{Alq}_{3}$ surfaces [13].
Our approach exploits this fact by depositing magnetic nanodots instead of isolated magnetic atoms on top of the organic layer to minimize the negative effects of the illdefined organic layer without the additional complications associated with an insulating barrier layer. Specifically, we use a growth method called buffer layer assisted growth (BLAG) $[14,15]$ to form the top magnetic electrode in a vertical organic spin valve of $\mathrm{Co} / \mathrm{Alq}_{3} / \mathrm{LSMO}$. The transport properties of BLAG prepared spin valves which exhibit sharper interfaces are compared to organic spin valves prepared through conventional atomic deposition of Co on $\mathrm{Alq}_{3}$, and a giant MR of up to $\sim 300 \%$ has been observed. In addition to achieving very large MR, junctions prepared using this method also maintain a simple trilayer structure which allows us to study the mechanism of the large MR without the complication of additional oxide layers on the interface.

LSMO thin films epitaxially grown by pulsed laser deposition on $\mathrm{SrTiO}_{3}$ (001) substrates were fabricated into bottom electrodes using conventional wet-etch optical lithography $[16,17]$. Both the BLAG and conventional spin valves are layered on the same single-crystal LSMO electrode then covered with the same $\mathrm{Alq}_{3}$ film thermal deposition for consistency (Fig. 1). For BLAG junctions, cobalt nanodots were grown on the $\mathrm{Alq}_{3}$ layers using the BLAG method $[14,15]$. On top of the Co nanodot layer, $\sim 7 \mathrm{~nm} \mathrm{Co}$ films were subsequently grown to complete the formation of Co electrode. Finally, a gold capping layer of $\sim 6 \mathrm{~nm}$ was grown and patterned into a crossbar configuration with respect to the bottom LSMO electrode using shadow masks. For conventional junctions, Co electrodes were formed by directly depositing Co onto the $\mathrm{Alq}_{3}$ layers. Because of the fact that the surface morphology of the $\mathrm{Alq}_{3}$ layer is not molecularly flat, we use effective thick- 




FIG. 1 (color online). (a) Schematic diagrams of a BLAG spin valve and a conventional spin valve. Cross-sectional schematic diagram of the conventional device (b) indicates the short circuit area (sketched by solid white line) due to the diffusion of Co atoms. For the BLAG device (c), several layers of Co nanodots (average nanodot volume $\sim 3.3 \mathrm{~nm}^{3}$ ) are formed using BLAG prior to the deposition of top electrodes which effectively minimizes the interdiffusion process.

ness $\left(d_{\text {eff }}=\right.$ thickness of the wetting layer, calibrated by atomic force microscope images) rather than the nominal thickness of the organic layers to denote spin valve thickness. The active device area is about $1 \mathrm{~mm} \times 2 \mathrm{~mm}$. The magnetoresistance (MR) is defined as $\mathrm{MR}=\left(R_{A P}-\right.$ $\left.R_{P}\right) / R_{A P}[18,19]$, where $R_{A P}$ and $R_{P}$ are the junction resistance measured when the magnetization of the Co and the LSMO electrodes are antiparallel and parallel, respectively.

Figure 2 compares MR values measured at $10 \mathrm{~K}$ for BLAG and conventional spin valves across a range of effective $\mathrm{Alq}_{3}$ layer thicknesses. For the BLAG spin valves, a negative MR of $\sim 1 \%\left(d_{\text {eff }}=23 \mathrm{~nm}\right)$ and $\sim 7 \%$ $\left(d_{\text {eff }}=67 \mathrm{~nm}\right)$ can be seen in Figs. 2(a) and 2(c), respectively. In contrast, conventional (direct atomic deposition of Co) junctions show no MR at $d_{\text {eff }}=23 \mathrm{~nm}$ [short circuit occurred, as Fig. 1(b)] and a much smaller negative MR of less than $1 \%$ at $d_{\text {eff }}=67 \mathrm{~nm}$ [Figs. 2(b) and 2(d)]. Moreover, the BLAG spin valves consistently exhibit larger resistances than the conventional devices, indicating that significantly less interdiffusion between the Co electrode and the $\mathrm{Alq}_{3}$ layer in the BLAG spin valves than in the conventional spin valves. Our resistance values are comparable to reported resistance of junctions with similar size that consist even thicker $\mathrm{Alq}_{3}$ layers $(100 \mathrm{~nm}$ to $300 \mathrm{~nm}$ of $\mathrm{Alq}_{3}$ ) and additional insulating $\mathrm{Al}_{2} \mathrm{O}_{3}$ layers [10]. This indicates that our BLAG method prevents interdiffusion at least as effectively as $\mathrm{Al}_{2} \mathrm{O}_{3}$. With increasing thickness of the $\mathrm{Alq}_{3}$ layer, the MR values increase substantially for both BLAG and conventional spin valves. In particular, at $d_{\text {eff }}=93 \mathrm{~nm}$, the BLAG spin valve exhibits the highest MR ever reported in an $\mathrm{Alq}_{3}$ based organic spin valve. A remarkable negative MR of $\sim 200 \%$ can be seen in Fig. 2(e). At the same effective thickness, the negative MR value of the conventional spin valve is $\sim 12 \%$, which is dramatically lower than that of the BLAG spin valves though consistent with previously reported values.


(e)

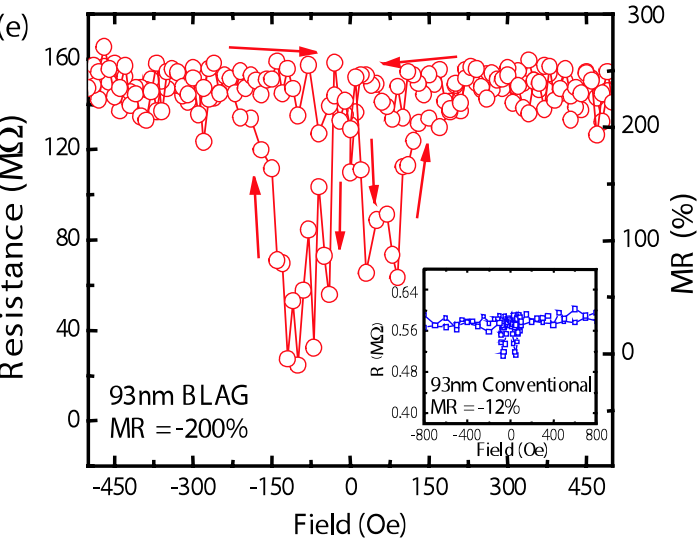

FIG. 2 (color online). Comparison of the MR loop response for junctions with a lower effective $\mathrm{Alq}_{3}$ thickness prepared by BLAG (a),(c) and conventional methods (b),(d), at a bias current of $-3 \mu$ A. (e) MR loop of the effective $\mathrm{Alq}_{3}$ thickness $(93 \mathrm{~nm}$ ) for BLAG junctions at a bias voltage of $-0.5 \mathrm{~V}$ shows giant MR response (inset: the MR loop of a conventional junction at the same effective thickness and bias voltage). The measurements were all taken at $10 \mathrm{~K}$.

The bias voltage dependence of the MR effect (calculated by $I-V$ curves between antiparallel and parallel configurations) for the BLAG and conventional junctions with effective $\mathrm{Alq}_{3}$ thicknesses of 23, 67, and $93 \mathrm{~nm}$ are shown in Figs. 3(a)-3(c), respectively. In general, MR values show a tendency to increase with decreasing bias voltage, although this increase is very small in conventional spin valves at lower spacer thicknesses. For the $93 \mathrm{~nm}$ conventional junction, MR reaches a maximum value of $\sim 35 \%$ at $-0.1 \mathrm{~V}$. This trend of increasing MR with decreasing bias is much more pronounced for the $93 \mathrm{~nm}$ BLAG junction. At $-0.3 \mathrm{~V}$, MR reaches $\sim 300 \%$. At biases smaller than those presented, the device resistance becomes too large to perform measurements in our setup. However, judging from the bias dependence of MR for all junctions, it can be expected that the MR values for the $93 \mathrm{~nm}$ thickness would greatly exceed $300 \%$ at lower bias voltages. The 



FIG. 3 (color online). Bias voltage dependence of MR ratio measured at $10 \mathrm{~K}$ comparison of conventional junctions and BLAG junctions with different $\mathrm{Alq}_{3}$ effective thicknesses: $23 \mathrm{~nm}$ (a), $67 \mathrm{~nm}$ (b), and $93 \mathrm{~nm}$ (c). (d) MR measured as a function of effective thickness for the two types of junctions.

asymmetric behavior of the bias dependence of MR has also been observed in both organic [1] and inorganic tunneling junctions [20]. Figure 3(d) shows the observed MR values of the two types of junctions as a function of effective thickness of the $\mathrm{Alq}_{3}$ spacer layer. An enhanced MR is observed at every thickness when the BLAG method is used. For both BLAG and conventional junctions, MR increases with increasing thickness from 23 to $93 \mathrm{~nm}$. This trend is different from recent reports that show decreasing MR with increasing insulating layer thickness [21,22], indicating that even the BLAG junctions are not completely free from the influence of the ill-defined layer when the $\mathrm{Alq}_{3}$ layer thickness is low, even though they exhibit much better properties than those of the conventional junctions. The effect of the ill-defined layer, however, decreases with increasing $\mathrm{Alq}_{3}$ layer thickness, leading to an increased MR. Above a certain thickness, spin-dependent transport is expected to decline due to the effect of the spin diffusion length inherent in the organic spacer layer; this behavior is observed as layer thickness surpasses the $93 \mathrm{~nm}$ thickness and shows a substantial MR reduction for the $135 \mathrm{~nm}$ thickness.

With the improved interface in the BLAG junction, we can now discuss the transport mechanism in organic spin valves by analyzing the measured current density-voltage $(J-V)$ characteristics. Figure 4 shows $J-V$ and $d I / d V$ curves for the 23,67, and $93 \mathrm{~nm}$ BLAG junction measured in both parallel and antiparallel magnetization configurations. The $J-V$ curve is quasilinear for the $23 \mathrm{~nm}$ junction but nonlinear for the 67 and $93 \mathrm{~nm}$ junctions. The $d I / d V$ curve of the $93 \mathrm{~nm}$ junction shows nearly parabolic bias dependence with no indication of a zero bias anomaly. For the lower effective thickness BLAG junctions, we expect low-mobility Ohmic conduction and space charge limited current (SCLC) to dominate over the injected charge contribution at low electric fields [23,24]. In this case, the current density is described by $J \propto V^{m}$, with $m \approx 1$ and $m \approx 1.25$ for the 23 and $67 \mathrm{~nm}$ junctions, respectively.

However, for the $93 \mathrm{~nm}$ junction, the fitted value of $m \approx$ 4 is too high in the trapped-charge-limited regime or SCLC for the low voltage used in our measurements $[25,26]$. In viewing of this discrepancy, we attempted to use tunneling based models to fit the data. Neither the standard FowlerNordheim (FN) tunneling type equation with the SchottkyNordheim barrier [27] nor the standard Simmons' tunneling model [28] yields satisfying results (not shown here).

Therefore, we turned back to the SCLC model, with a modification that includes a screened Frenkel effect. The Frenkel effect is likely observed when the field strength is greater than $1.0 \mathrm{kV} / \mathrm{cm}$ at low temperatures before the trap-filled-limited voltage is reached [29]. However, we found that the unscreened Frenkel model overestimates the curvature of our measured $J-V$ curve by several orders of magnitude. If we consider that the potential of the ion trap centers is screened, the $J-V$ relationship can be obtained approximately as follows [30]:

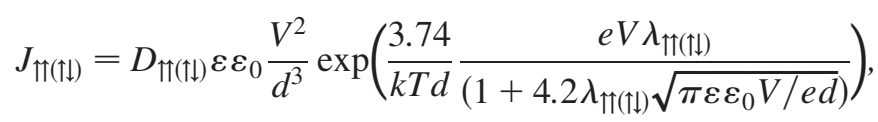
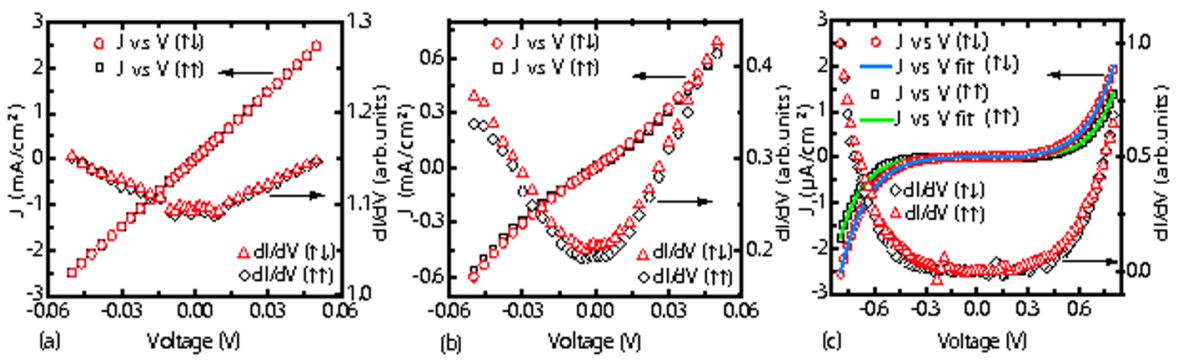

FIG. 4 (color online). Current density-voltage $(J-V)$ and $(d I / d V)$ characteristics for the BLAG junction with effective thickness $\mathrm{Alq}_{3}$ spacer layers of $23 \mathrm{~nm}$ (a), $67 \mathrm{~nm}$ (b), and $93 \mathrm{~nm}$ (c) in antiparallel and parallel configurations. Experimental data for $93 \mathrm{~nm}$ junction at both antiparallel configuration ( $-40 \mathrm{Oe}$ ) and parallel configuration ( $-190 \mathrm{Oe}$ ) are fitted using Eq. (1) (solid lines). 
TABLE I. Fitting parameters used for junctions created using the BLAG and conventional methods denoted by (BLAG) and (conv), respectively, with corresponding spin-dependent injection MR.

\begin{tabular}{lccccrr}
\hline \hline$d(\mathrm{~nm})$ & $D_{\Uparrow}$ & $N_{\text {tot }(\uparrow)}\left(\mathrm{cm}^{-3}\right)$ & $D_{\Uparrow \downarrow}$ & $N_{\text {tot }(\uparrow \downarrow)}\left(\mathrm{cm}^{-3}\right)$ & $D_{\uparrow \downarrow} / D_{\Uparrow}$ & $\mathrm{MR}$ \\
\hline 93 (BLAG) & $9.1688 \mathrm{E}-19$ & $1.45379 \mathrm{E} 19$ & $3.9472 \mathrm{E}-18$ & $2.77563 \mathrm{E} 19$ & 4.31 & 300 \\
93 (conv) & $3.8752 \mathrm{E}-16$ & $3.41038 \mathrm{E} 19$ & $8.3410 \mathrm{E}-16$ & $5.90169 \mathrm{E} 19$ & 2.15 & 35 \\
135 (BLAG) & $2.0580 \mathrm{E}-15$ & $4.25549 \mathrm{E} 19$ & $2.2035 \mathrm{E}-15$ & $4.68603 \mathrm{E} 19$ & 1.07 & 13 \\
135 (conv) & $1.4661 \mathrm{E}-15$ & $4.33485 \mathrm{E} 19$ & $1.4790 \mathrm{E}-15$ & $4.37145 \mathrm{E} 19$ & 1.01 & 4 \\
\hline \hline
\end{tabular}

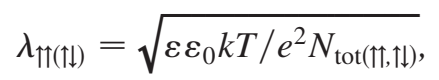

where $\lambda$ is the screening length of the medium, $e$ is the electron charge, $k$ is the Boltzmann constant, $T$ is the temperature, $\varepsilon$ is the dielectric constant, $d$ is the thickness of the organic layer, $N_{\text {tot }(\uparrow \uparrow, \uparrow \downarrow)}$ is the total density with the sum of free and trapped charges, and $D_{\uparrow \uparrow(\uparrow))}$ is a spindependent prefactor that depends on the antiparallel ( $\uparrow \downarrow$ ) and parallel ( $\uparrow$ ) magnetization configurations and combines the effect of the effective interfacial area and the carrier injection efficiency. Note that there are only two $(D$ and $\lambda$ ) fitting parameters for each $J-V$ curve.

The fitted $J-V$ curves using Eq. (1) for the $93 \mathrm{~nm}$ thick BLAG device is shown in Fig. 4(c) for both antiparallel and parallel configurations. The Frenkel effect with the screened potential is observable when the voltage is above $0.3 \mathrm{~V}$, at which the exponent $\frac{4.2}{k T d} \frac{e^{2} \lambda V}{\left(1+2.1 \sqrt{\left.4 \pi \varepsilon \varepsilon_{0} V / e \lambda d\right)}\right.}$ is of order unity or greater [30]. Table I shows the fitted parameters for $J-V$ curves from our samples. The values of the Frenkel factor $\left[F_{F(\uparrow \uparrow \uparrow \downarrow)}\right.$, see Ref. [30] ] are generally larger for samples without BLAG, reflecting possible interdiffusion between the Co electrode and the $\mathrm{Alq}_{3}$ spacer layer that leads to larger contact areas. The values of $N_{\text {tot }}$ are consistent with the published results $\left[\sim 10^{19} / \mathrm{cm}^{3}\right.$, Ref. [25] ]. The huge difference in the prefactors $D_{\uparrow \downarrow(\uparrow)}$ between the two spin configurations is consistent with the measured MR. This result strongly suggests that spindependent carrier injection is the main cause of the observed large MR.

This work provides a generic approach to fundamentally solve the bottleneck problem in studying organic spin valves, i.e., the ill-defined interface between magnetic electrodes and the organic spacer layer. Using the BLAG method, organic spin valves with greatly improved interfaces can be consistently fabricated which allows for higher MR and a cleaner system in which to study the fundamental phenomena driving spin transfer in organic devices.

This effort was supported by the U.S. DOE Office of Basic Energy Sciences, Division of Materials Science and Engineering, through Oak Ridge National Laboratory, D. S. acknowledges partial support from Chinese Academy of Sciences. Z. G. and X.-G.Z. acknowledge partial support from Oak Ridge National Laboratory's
Center for Nanophase Materials Sciences, sponsored by the Scientific User Facilities Division, Office of Basic Energy Sciences, U.S. Department of Energy.

*To whom correspondence should be addressed. shenj@ornl.gov

[1] Z.H. Xiong et al., Nature (London) 427, 821 (2004).

[2] F. J. Wang et al., Synth. Met. 155, 172 (2005).

[3] S. Majumdar et al., Appl. Phys. Lett. 89, 122114 (2006).

[4] H. Vinzelberg et al., J. Appl. Phys. 103, 093720 (2008).

[5] S. Pramanik et al., Nature Nanotech. 2, 216 (2007).

[6] W. Xu et al., Appl. Phys. Lett. 90, 072506 (2007).

[7] J. S. Jiang, J. E. Pearson, and S. D. Bader, Phys. Rev. B 77, 035303 (2008).

[8] T. S. Santos et al., Phys. Rev. Lett. 98, 016601 (2007).

[9] J. H. Shim et al., Phys. Rev. Lett. 100, 226603 (2008).

[10] V. Dediu et al., Phys. Rev. B 78, 115203 (2008).

[11] Y. Q. Zhan et al., Appl. Phys. Lett. 94, 053301 (2009).

[12] Jung-Woo Yoo et al., Phys. Rev. B 80, 205207 (2009).

[13] J. H. Lee, Yeonjin Yi, and D. W. Moon, Appl. Phys. Lett. 93, 153307 (2008).

[14] L. Huang, S. J. Chey, and J. H. Weaver, Phys. Rev. Lett. 80, 4095 (1998).

[15] J. P. Pierce et al., Phys. Rev. Lett. 92, 237201 (2004).

[16] J. X. Ma et al., Phys. Rev. Lett. 95, 237210 (2005).

[17] H. Y. Zhai et al., Phys. Rev. Lett. 97, 167201 (2006).

[18] M. Julliere, Phys. Lett. A 54, 225 (1975).

[19] J. S. Moodera et al., Phys. Rev. Lett. 74, 3273 (1995).

[20] J. M. De Teresa et al., Science 286, 507 (1999).

[21] A. J. Drew et al., Nature Mater. 8, 109 (2009).

[22] V. Dediu et al., Nature Mater. 8, 707 (2009).

[23] M. A. Lampert and P. Mark, Current Injection in Solids (Academic, New York, 1970).

[24] V. I. Arkhipov, H. von Seggern, and E. V. Emerlianova, Appl. Phys. Lett. 83, 5074 (2003).

[25] P. E. Burrows et al., J. Appl. Phys. 79, 7991 (1996).

[26] A. Loannidis et al., Appl. Phys. Lett. 72, 3038 (1998).

[27] M. Pope and C.E. Swenberg, Electronic Process in Organic Crystals and Polymers (Oxford University Press, Oxford, 1999), 2nd ed.

[28] J. G. Simmons, J. Appl. Phys. 34, 1793 (1963).

[29] P. N. Murgatroyd, J. Phys. D 3, 151 (1970), and references therein.

[30] X.-G. Zhang (unpublished); see supplementary material at http://link.aps.org/supplemental/10.1103/ PhysRevLett.104.236602 for theory details. 